Trinity University

Digital Commons @ Trinity

3-2018

Evaluating Differences in the Active-Site Electronics of Supported Au Nanoparticle Catalysts Using Hammett and DFT Studies

G. Kumar

Luke Tibbitts

Jaclyn Newell

Basu Panthi

Ahana Mukhopadhyay

See next page for additional authors

Follow this and additional works at: https://digitalcommons.trinity.edu/chem_faculty

Part of the Chemistry Commons 


\section{Authors}

G. Kumar, Luke Tibbitts, Jaclyn Newell, Basu Panthi, Ahana Mukhopadhyay, Robert M. Rioux, Christopher J. Pursell, M. Janik, and Bert D. Chandler 


\title{
Evaluating differences in the active-site electronics of supported Au nanoparticle catalysts using Hammett and DFT studies
}

\author{
Gaurav Kumar', Luke Tibbitts', Jaclyn Newell2, Basu Panthi², Ahana Mukhopadhyay', \\ Robert M. Rioux ${ }^{1,3}$, Christopher J. Pursell ${ }^{2}$, Michael Janik ${ }^{1}$ and Bert D. Chandler ${ }^{2 \star}$
}

\begin{abstract}
Supported metal catalysts, which are composed of metal nanoparticles dispersed on metal oxides or other high-surface-area materials, are ubiquitous in industrially catalysed reactions. Identifying and characterizing the catalytic active sites on these materials still remains a substantial challenge, even though it is required to guide rational design of practical heterogeneous catalysts. Metal-support interactions have an enormous impact on the chemistry of the catalytic active site and can determine the optimum support for a reaction; however, few direct probes of these interactions are available. Here we show how benzyl alcohol oxidation Hammett studies can be used to characterize differences in the catalytic activity of Au nanoparticles hosted on various metal-oxide supports. We combine reactivity analysis with density functional theory calculations to demonstrate that the slope of experimental Hammett plots is affected by electron donation from the underlying oxide support to the Au particles.
\end{abstract}

$\square$ eterogeneous catalysts are widely used in the chemical industry, particularly in petroleum refining, bulk chemicals production, pharmaceuticals and fine chemicals synthesis, and environmental remediation ${ }^{1}$. Supported metal nanoparticle catalysts, which consist of an active metal component dispersed on a high-surface-area solid, are one of the most important classes of industrial catalyst ${ }^{1}$. Our ability to control and tune catalysts is impacted by our ability to identify and understand the factors that control the chemistry at the active $\operatorname{site}^{2-4}$. Numerous strategies have been employed to improve catalyst reactivity. For example, simply changing the underlying support may influence the morphology of metal nanoparticles ${ }^{5}$ and associated electronics ${ }^{5-9}$; this, in turn, can have significant effects on catalytic activity ${ }^{10-13}$ and selectivity ${ }^{14}$. Identifying and characterizing catalytic active sites, however, remains difficult for supported nanoparticle catalysts because these materials are inherently non-uniform ${ }^{15}$. This presents substantial challenges to understanding the variables that give rise to the chemistry occurring at the active site.

The electronic interactions between a metal nanoparticle and its oxide support are important, but complicated. Charge transfer between the two components can occur in either direction depending on the metal, the reducibility of the support, the exposed metal facets and the presence of defects ${ }^{5-9,16-18}$. There are few good methods for evaluating how these potential effects might influence the catalysis. Here, we report the use of a Hammett probe reaction to directly measure electronic differences in the catalytic active site of supported Au nanoparticles. We use this reactivity analysis as a catalyst descriptor along with density functional theory (DFT) studies to demonstrate that the experimental Hammett slopes correlate with the ability of the underlying oxide support to donate charge to the metal particle.

Haruta's seminal discovery of low-temperature CO oxidation activity over supported $\mathrm{Au}$ nanoparticles ${ }^{19}$ sparked a wave of exploration into gold catalysis ${ }^{20}$. Although non-aerobic processes have been investigated ${ }^{21,22}$, the majority of studies have focused on CO oxidation $19,20,23,24$, organic oxidations ${ }^{25-27}$ and oxidative couplings ${ }^{28,29}$. Selective alcohol oxidations have been of particular interest due to the high selectivity for aldehydes and ketones. Benzyl alcohol (BA) selective oxidation has been particularly well studied $^{13,30,31}$. Three elementary steps may impact the BA oxidation rate over Au catalysts: (1) substrate adsorption onto the support, presumably through hydrogen-bonding interactions between the alcohol and support hydroxyls, (2) proton transfer from the alcohol to the support yielding a more reactive alkoxide, and (3) hydride transfer from the benzylic carbon to the Au nanoparticle. Strong evidence that the hydride transfer step is rate-determining was demonstrated by kinetics studies over $\mathrm{Au} / \mathrm{CeO}_{2}$ (ref. 32) and $\mathrm{Au} / \mathrm{TiO}_{2}$ (ref. 33) catalysts, which reported a primary kinetic isotope effect (KIE) for the benzylic $\mathrm{C}-\mathrm{H}$. Hammett studies confirmed the build-up of positive charge on the benzylic carbon in the rate-determining step (RDS), which was further supported by spin-trapping experiments ${ }^{34}$

The underlying support has significant effects on many Aucatalysed reactions, particularly $\mathrm{BA}$ oxidation $^{30,32}$. Because the RDS involves hydride transfer from the benzylic carbon to $\mathrm{Au}$ (Fig. 1), we hypothesize that support effects are related to the electron richness of the Au surface. This is a particularly difficult catalyst property to evaluate and we are unaware of experimental methods that directly probe subtle differences in the surface electronics of reaction centres during a reaction.

Hammett reactivity studies are classic tools for evaluating charge transfer during a reaction, and they provide a potential new probe for catalyst electronic effects ${ }^{35}$. Hammett studies are typically performed by substituting the para position of a phenyl ring and measuring differences in the reaction rates. The electron-donating or -withdrawing ability of the substituent is quantified by its

'Department of Chemical Engineering, The Pennsylvania State University, University Park, Pennsylvania 16802-4400 USA. ${ }^{2}$ Department of Chemistry, Trinity University, San Antonio, Texas 78212-7200, USA. ${ }^{3}$ Department of Chemistry, The Pennsylvania State University, University Park, Pennsylvania 16802-4400, USA. *e-mail: bert.chandler@trinity.edu 


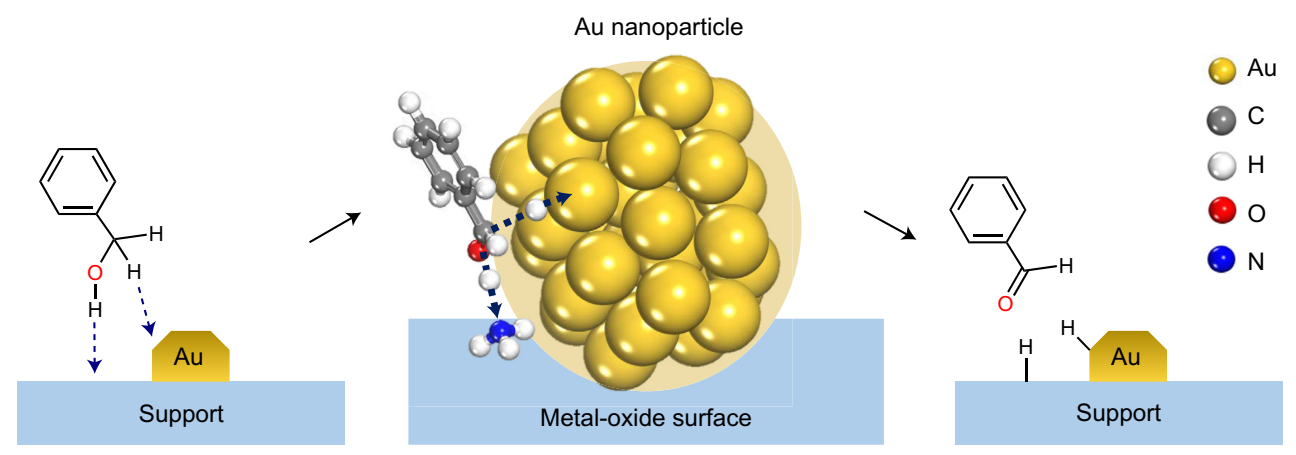

Figure 1 | Reaction schematic of BA oxidation over a metal-oxide-supported Au nanoparticle. BA and the Au/support form the initial state (left) and benzaldehyde and the Au-H/protonated support are the final state (right). The transition state (middle) shows a concerted hydride transfer (to the Au nanoparticle) and proton transfer (to the oxide support). $\mathrm{NH}_{3}$ acts as a model for the proton-accepting support in the DFT calculations.

substituent constant or sigma $(\sigma)$ value. Sigma is defined by the effect of the substituent $(x)$ on the benzoic acid dissociation constant in water $\left(\sigma_{x}=\log \left(K_{x} / K_{\mathrm{BA}}\right)\right)$. When the logarithm of the reaction rate varies directly with $\sigma$, a linear free energy relationship (LFER) is said to exist. The reactivity differences are interpreted in terms of changing electron richness/poorness of the benzylic carbon and are commonly used as a semiquantitative evaluation of the charge build-up at the benzylic carbon in the reaction transition state ${ }^{36}$.

The Corma ${ }^{32}$ and Fristrup ${ }^{33}$ groups previously demonstrated that BA oxidation rates are linearly correlated with $\sigma^{+}\left(\sigma^{+}\right.$values are derived from a slightly different reference reaction to account for resonance stabilization effects when a positive charge develops in the transition state $)^{36}$. The negative slope of this line, defined as $\rho$, indicates a build-up of positive charge on the benzylic carbon in the RDS transition state. This was key mechanistic evidence that hydride transfer to the $\mathrm{Au}$ surface was rate-determining (middle image, Fig. 1$)^{32,33}$. Based on these results, we reasoned that a series of carefully performed Hammett studies would provide a new method for describing differences in catalyst active sites and could be a useful tool for studying supported-nanoparticle surface electronics.

\section{Results}

We compared a series of $1 \mathrm{wt} \%$ Au catalysts supported on several different oxide supports $\left(\mathrm{Au} / \mathrm{Al}_{2} \mathrm{O}_{3}, \mathrm{Au} / \mathrm{SiO} \mathrm{O}_{2}, \mathrm{Au} / \mathrm{TiO}_{2}\right.$ and $\mathrm{Au} / \mathrm{ZnO}$ ). Turnover frequencies (TOFs, Table 1) were determined using kinetic thiol poisoning titrations to estimate the number of active sites ${ }^{37}$. Briefly, the reaction rate was measured as a function of substoichiometric amounts of added thiol. Extrapolating plots of rate versus thiol added to zero activity and assuming a thiol: active site stoichiometry of unity estimated the number of active sites $^{37}$. The resulting TOFs vary by a factor of two, indicating a clear support effect on the catalytic activity of the reaction centres.

Hammett studies. Figure 2a shows a complete Hammett study using a $3 \mathrm{wt} \% \mathrm{Au} / \mathrm{Al}_{2} \mathrm{O}_{3}$ catalyst, plotting the log of the reaction rate versus $\sigma^{+}$. As Corma ${ }^{32}$ and Fristrup ${ }^{33}$ showed, BA oxidation correlates far better with $\sigma^{+}$than $\sigma$ (Supplementary section 'Substituents and substituent constants'). The negative slope of the line in Fig. 2a (defined as $\rho_{\text {obs }}$ for that catalyst) indicates that the RDS transition state develops a partial positive charge on the benzylic carbon that is resonance stabilized by electron-donating substituents on the phenyl ring ${ }^{36}$. This is entirely consistent with aforementioned mechanistic studies that implicate hydride transfer $\mathrm{Au}$ as the $\mathrm{RDS}^{32-34}$

Our initial studies examined four benzyl alcohols (BAs and parasubstituted $-\mathrm{OCH}_{3},-\mathrm{CH}_{3}$ and $-\mathrm{CF}_{3}$ ) over the four $1 \% \mathrm{Au}$ catalysts. The rate for unsubstituted benzoic acid (or the $-\mathrm{H}$ substituent) was consistently faster than predicted by the Hammett relationship for the substituted BAs (Supplementary section 'Substituents and substituent constants'). We therefore performed an additional study that included $-\mathrm{Cl}$ and $-{ }^{t} \mathrm{Bu}$ substituted BA (Fig. 2a). The $-\mathrm{Cl}$ substituted $\mathrm{BA}$ is consistent with the trend for other similarly sized substituents $\left(-\mathrm{OCH}_{3},-\mathrm{CH}_{3}\right.$ and $\left.-\mathrm{CF}_{3}\right)$. Unsubstituted $\mathrm{BA}$ and the larger $-{ }^{t} \mathrm{Bu}$ substituted $\mathrm{BA}$ showed opposite trends, indicating that sterics may play an important role in surface reactions.

Hammett studies are traditionally used for solution-phase reactions where both reacting species are generally free to adopt orientations that minimize steric interactions. For a heterogeneously catalysed reaction, however, the Au nanoparticle is immobilized on a support. BA mobility is also constrained by its adsorption on the support through the alcohol group. Consequently, for BA oxidation to occur, the support-adsorbed BA must approach the catalytic active site with the phenyl ring largely parallel to the surface of the Au nanoparticle. Relative to a solution-phase reaction, this forces greater steric interactions between the phenyl ring substituents and the Au surface.

To better illustrate these effects, we calculated the distance to which each substrate can approach a $\mathrm{Au}(111)$ surface, using the optimized substrate geometry and constraining the phenyl ring to be parallel to the surface. The approach distance $(d$, table in

Table 1 | Experimentally observed TOFs for BA oxidation; Hammett slope $\rho$; average particle size; isoelectric point; XPS O 1s, XPS Au $4 d / 4 f$ and oxide bandgaps for $\mathrm{Au} / \mathrm{Al}_{2} \mathrm{O}_{3}, \mathrm{Au} / \mathrm{SiO}_{2}, \mathrm{Au} / \mathrm{TiO}_{2}$ and $\mathrm{Au} / \mathrm{ZnO}$.

\begin{tabular}{|c|c|c|c|c|c|c|c|c|}
\hline \multirow[t]{2}{*}{ Catalyst } & \multirow{2}{*}{$\begin{array}{l}\text { TOF (mol per } \\
\text { site per min) }\end{array}$} & \multirow[t]{2}{*}{$\rho$} & \multirow{2}{*}{$\begin{array}{l}\text { Average particle } \\
\text { size }(\mathrm{nm})\end{array}$} & \multirow{2}{*}{$\begin{array}{l}\text { Support } \\
\text { bandgap }(e V)^{38}\end{array}$} & \multicolumn{3}{|l|}{ XPS (eV) } & \multirow{2}{*}{$\begin{array}{l}\text { Support } \\
\text { isoelectric point }{ }^{43}\end{array}$} \\
\hline & & & & & $A u(4 f)$ & $A u(4 d)$ & $O(1 s)$ & \\
\hline $1 \% \mathrm{Au} / \mathrm{Al}_{2} \mathrm{O}_{3}$ & $5.4 \pm 0.6$ & $-0.87 \pm 0.05$ & $2.5 \pm 0.7$ & $7 \pm 1$ & 84.0 and 87.5 & 334.1 and 354.4 & 531.3 & $7-9$ \\
\hline $1.5 \% \mathrm{Au} / \mathrm{SiO}_{2}$ & $5.3 \pm 0.6$ & $-0.83 \pm 0.06$ & $3.5 \pm 0.7$ & $8.9 \pm 0.1$ & 83.9 and 87.4 & $-^{a}$ & 532.7 & $2-3$ \\
\hline $1 \% \mathrm{Au} / \mathrm{TiO}_{2}$ & $3.4 \pm 0.4$ & $-0.44 \pm 0.02$ & $2.9 \pm 0.7$ & $3.3 \pm 0.4$ & 80.8 and 84.4 & 337.3 and 356.1 & 529.7 & $5-7$ \\
\hline $1 \% \mathrm{Au} / \mathrm{ZnO}$ & $2.8 \pm 0.3$ & $-0.36 \pm 0.03$ & $3.0 \pm 0.9$ & $3.3 \pm 0.1$ & $-\mathrm{b}$ & 335.5 and 352.5 & 530.3 & $9-10$ \\
\hline
\end{tabular}

No correlation is found between $\rho$ and particle size, support isoelectric point or XPS data; a weak correlation is found between $\rho$ and the oxide bandgaps. Reported errors are standard deviations from experimental measurements or-for support bandgaps-available literature values.

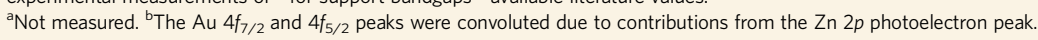


a
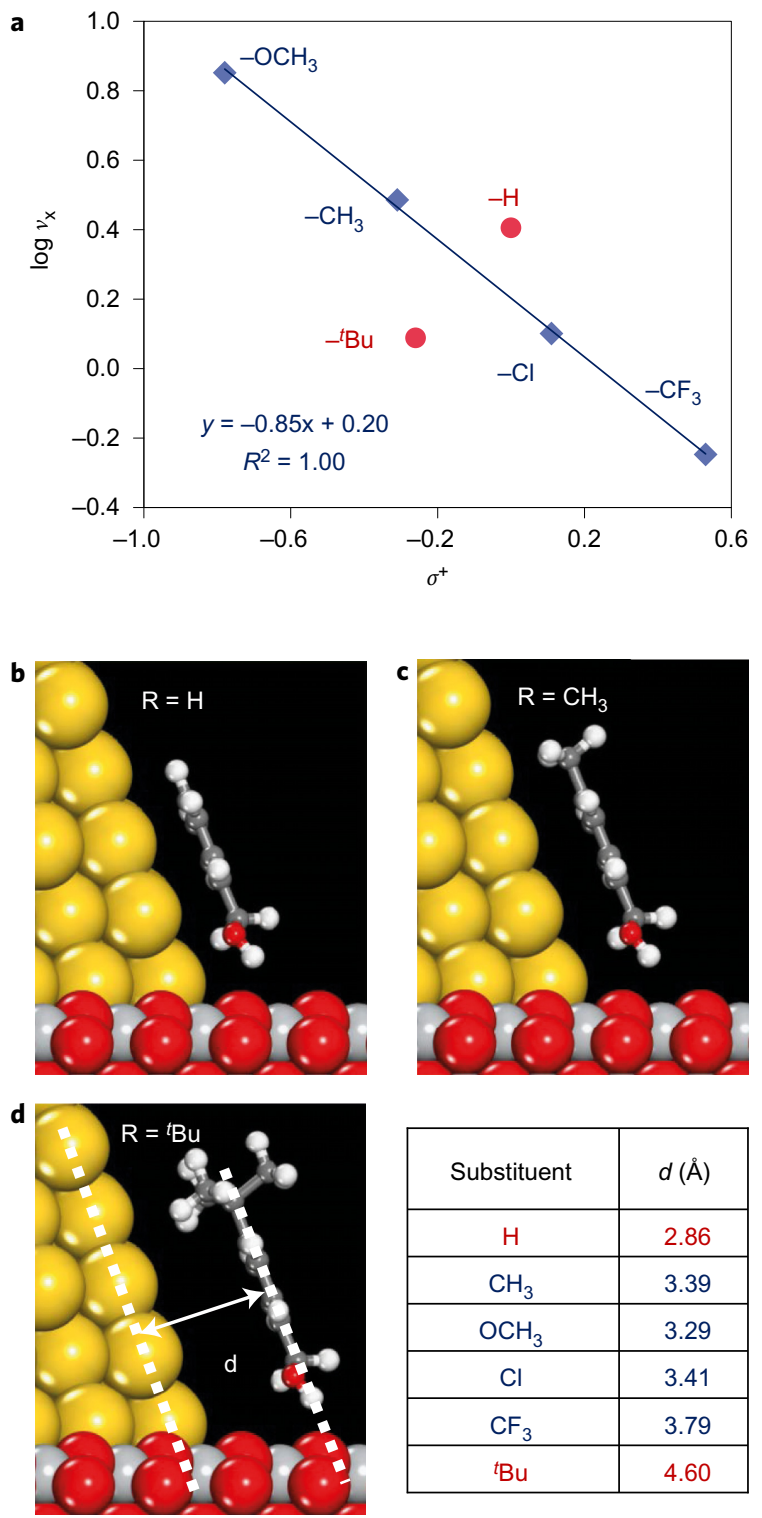

\begin{tabular}{|c|c|}
\hline Substituent & $d(\AA)$ \\
\hline $\mathrm{H}$ & 2.86 \\
\hline $\mathrm{CH}_{3}$ & 3.39 \\
\hline $\mathrm{OCH}_{3}$ & 3.29 \\
\hline $\mathrm{Cl}$ & 3.41 \\
\hline $\mathrm{CF}_{3}$ & 3.79 \\
\hline${ }^{\mathrm{B}} \mathrm{Bu}$ & 4.60 \\
\hline
\end{tabular}

Figure 2 | Substituent effects on BA oxidation. a, BA oxidation Hammett study for a $3 \mathrm{wt} \% \mathrm{Au} / \mathrm{Al}_{2} \mathrm{O}_{3}$ catalyst. For the sterically similar parasubstituted $\mathrm{BAs}\left(\mathrm{X}=-\mathrm{OCH}_{3},-\mathrm{CH}_{3},-\mathrm{Cl}_{1}-\mathrm{CF}_{3}\right)$, there is a linear relationship between the measured log of the reaction rate $\left(\nu_{x}\right)$ and the $\sigma^{+}$parameter. b-d, Depictions of the van der Waals approach distance between benzyl alcohols and the Au surface at the metal-support interface. The table shows how the ring substituents affect the approach of the alcohol to the catalyst active sites. Differences in this approach distance $d$ help to explain why the sterically smaller $(-\mathrm{H})$ and larger $\left(-{ }^{t} \mathrm{Bu}\right)$ substituents deviate from the linear Hammett relationship in a (see Supplementary section 'Substituents and substituent constants'). Au, gold; O, red; H, white; C, grey; Ti (support), grey.

Fig. 2) was then defined as the distance between the phenyl ring and $\mathrm{Au}(111)$ surface. The large $t$-butyl group clearly prevents the substrate from approaching the surface, while the $-\mathrm{CH}_{3},-\mathrm{OCH}_{3},-\mathrm{Cl}$ and $-\mathrm{CF}_{3}$ substituents have comparable approach distances. The unsubstituted BA can approach slightly closer. Although this is a simplification of the support-nanoparticle interface, it clearly illustrates the importance of the substituent sterics in this reaction. Based on the reactivity data and this analysis, we did not include the $-\mathrm{H}$ or $-{ }^{t} \mathrm{Bu}$ substituents in the Hammett studies used to describe the $1 \%$ Au catalysts (Fig. 3a). Instead, the unsubstituted BA activity was used as a separate measurement for comparing the Hammett trends over the various metal oxide supported Au catalysts (Fig. 3b). We note that including unsubstituted BA in the Hammett analysis does not change any of the determined slopes by more than 0.02 , so this exclusion has no effect on the overall conclusions of the study.

The $\rho_{\text {obs }}$ values (Fig. 3a) range from -0.36 for $\mathrm{Au} / \mathrm{ZnO}$ to -0.87 for $\mathrm{Au} / \mathrm{Al}_{2} \mathrm{O}_{3}$, indicating that Hammett studies probe subtle differences in catalytic active sites and that the underlying support significantly influences the active-site electronics. For comparison, if the supports had no influence on the transition-state energy, the $\rho$ values would be the same and the lines in Fig. 3a would be parallel. To express these relationships in terms of traditional LFERs, each catalyst can be referenced back to the original $\sigma^{+}$parameters:

$$
\log \left(\frac{k_{x, \mathrm{cat}}}{k_{\mathrm{H}, \mathrm{cat}}}\right)=\rho_{\text {obs }} \sigma_{\text {substituent }}^{+}
$$

Because each catalyst shows a LFER with the $\sigma^{+}$values, differences in $\rho_{\text {obs }}$ can be directly attributed to differences in the ability of the catalyst to stabilize the hydride transfer transition state (see DFT studies below). Furthermore, because these Hammett studies directly probe hydride transfer to the active site on Au during the RDS, they are not influenced by other reaction parameters such as the number of active sites on the catalyst.

Fundamentally, the Hammett parameter $\rho$ measures the reaction's sensitivity to subtle differences in substrate electronics. This is traditionally interpreted in terms of stabilization of charge build-up in the transition state of the RDS. During hydride transfer, any positive charge that builds on the benzylic carbon must be compensated by a combination of negative charge on the Au and loss of negative charge on the alkoxide. It follows that the reaction rate will be affected by both the substituent and the catalyst's ability to stabilize the developing hydride. Consequently, for these reactions, the experimental $\rho_{\text {obs }}$ values are simultaneously influenced by two similar electronic effects: (1) the substituent's ability to help to stabilize or destabilize the positive charge on the benzylic carbon and (2) the catalyst's ability to stabilize negative charge on the Au. The experimental data clearly show the underlying support has an important influence on this sensitivity, with $\mathrm{Au} / \mathrm{Al}_{2} \mathrm{O}_{3}$ and $\mathrm{Au} / \mathrm{SiO}_{2}$ being very sensitive to differences in substrate electronics and $\mathrm{Au} / \mathrm{TiO}_{2}$ and $\mathrm{Au} / \mathrm{ZnO}$ being less sensitive.

Relationship between Hammett studies, BA oxidation activity and physical characterization data. The measured TOFs for unsubstituted BA closely correlate with $\rho_{\text {obs }}$ (Fig. 3b). Although this is not a LFER, the correlation shows BA operates by the same mechanism as the substituted reactants and is influenced by the same electronic effects. The primary difference is that $\mathrm{H}$ is smaller than the other substituents, which allow BA to more closely approach the $\mathrm{Au}$ nanoparticle, making $\mathrm{BA}$ slightly more reactive than the trend based on the larger substituents. This also means that the conclusions from the Hammett studies using substituted BAs can be directly applied to BA (that is, including BA in the Hammett plots results in no significant differences in the $\rho_{\text {obs }}$ values). For simplicity, further comparisons are therefore made with BA.

We sought to correlate the trend in BA reactivity and Hammett studies with characterization data from standard techniques (Table 1). First, the Au particle sizes are similar $(\sim 3 \mathrm{~nm})$, and $\rho_{\text {obs }}$ shows no correlation with the small variations in particle size. $\mathrm{Au} / \mathrm{SiO}_{2}$ and $\mathrm{Au} / \mathrm{Al}_{2} \mathrm{O}_{3}$ have similar $\rho_{\text {obs }}$ values yet have the largest and smallest particles in the study, respectively. A weak correlation exists between $\rho_{\text {obs }}$ and the literature bandgap values and the X-ray photoelectron spectroscopy (XPS) O(1s) peak position (Supplementary section 'Catalyst characterization data'), demonstrating that the Hammett studies report on a subtle, yet 
a

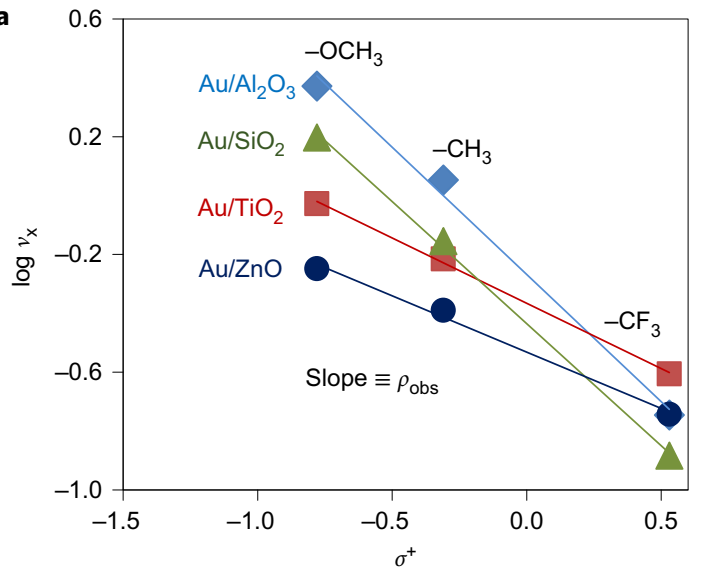

b

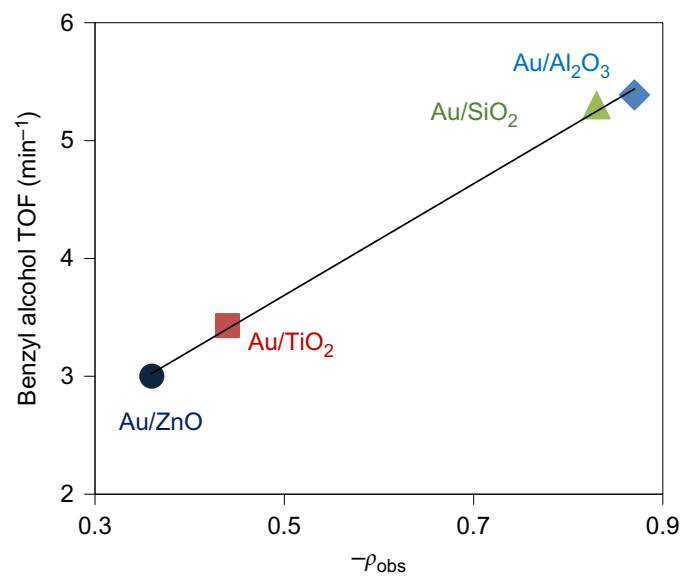

Figure 3 | Substituent and support effects on BA oxidation. $\mathbf{a}$, Hammett relationships for $\mathrm{Au} / \mathrm{Al}_{2} \mathrm{O}_{3}, \mathrm{Au} / \mathrm{SiO}_{2}, \mathrm{Au} / \mathrm{TiO}_{2}$ and $\mathrm{Au} / \mathrm{ZnO}$ ( 1 wt\% $\mathrm{Au}$ each). The slopes of the lines are referred to as the Hammett parameter $\rho$ for each catalyst. $\mathbf{b}$, Linear relationship between the BA turnover frequency (determined by thiol poisoning experiments ${ }^{37}$ ) and $\rho_{\text {obs }}$ (determined from substituted BAs in $\mathbf{a}$ ), showing a clear influence of the underlying support on both BA turnover frequency and $\rho_{\text {obs. }}$. Each data point shown is an average of at least two runs, and standard deviations are less than $\pm 12 \%$.

important, influence of the support on the Au nanoparticles. Correlations with the Au XPS data ( $4 d$ and $4 f$ peaks) are unclear.

These physical characterization methods are essentially bulk measurements for $3 \mathrm{~nm}$ Au nanoparticles. A significant advantage of the Hammett methodology is that it only probes the catalytic active sites. This is particularly important for materials where the active site may have a somewhat different electronic environment from even other surface atoms, such as atoms at the metal-support interface. These metal-support interface sites are often invoked in explaining the high activity of Au catalysts, so Hammett studies may be particularly useful in probing these systems.

DFT studies: Au particle charge effects. We applied DFT to firmly establish the relationship between the Hammett studies and the charge at the surface of the Au particle, hypothesizing that differences in $\rho_{\text {obs }}$ are due to differences in the electron richness of the Au active sites. These sites will be heavily influenced by the support, particularly if they are primarily at the metal-support interface. DFT modelling of the entire reaction pathway over Au particles on four model support structures was deemed too costly. We therefore investigated only the rate-limiting hydride transfer, using a 35-atom Au cluster $(\sim 1 \mathrm{~nm})$ to represent the $\mathrm{Au}$ nanoparticle in our DFT model. We note that $\mathrm{O}_{2}$ binding is thermodynamically unfavourable in this model (Supplementary section 'Computational data'), so we focused our analysis on the rate-determining hydride transfer step.

Initially, we wanted to isolate our DFT studies to probe only the effects of particle charge; however, attempts to model the reaction using only BA and the Au cluster were unsuccessful. We discovered that, while not rate-limiting, the weak Brønsted base functionality of the support is critical for this reaction. To simply and inexpensively incorporate this acid-base chemistry into the computational model, we included an $\mathrm{NH}_{3}$ molecule near the $\mathrm{Au}_{35}$ cluster. Note that the experimental results also show no correlation between $\rho$ and the support isoelectric point or the O $1 s$ XPS peak energy, indicating that the differences in $\rho$ do not arise from variations in support basicity. The DFT calculations (including $\mathrm{NH}_{3}$ ) successfully modelled the RDS as a hydride transfer to the Au cluster, coupling it to a proton transfer from the alcohol to ammonia (middle image, Fig. 1). The transition state has a localized partial positive charge on the benzylic carbon and a developing negative charge on the $\mathrm{Au}$, consistent with the experimental evidence.

We then varied the charge on the $\mathrm{Au}_{35}$ cluster to understand how Au surface electronics affect the hydride transfer. Hydride transfer reaction energies were calculated for BA and the para-substituted $\left(-\mathrm{CF}_{3}\right.$ and $\left.-\mathrm{OCH}_{3}\right)$ alcohols using three charged $\mathrm{Au}_{35}$ clusters. Several conclusions become immediately apparent from the transition state geometries (Supplementary section 'Computational data'). First, the energy and position of the transition state depend strongly on the Au cluster charge. The positively charged cluster has relatively early transition states, evidenced by short $\mathrm{C}-\mathrm{H}$ and long $\mathrm{Au}-\mathrm{H}$ interactions. Conversely, the negatively charged cluster goes through later transition states, in which the $\mathrm{C}-\mathrm{H}$ bond is almost completely broken.

Using several cluster charges $(\sim-2,0,2)$, the calculated activation barriers and reaction energies exhibit a Brønsted-Evans-Polanyi (BEP) relationship (Fig. 4a). Because this linear relation precludes the need for computationally demanding transition-state searches for all cluster charges, we used the reaction energies as a measure of reactivity in subsequent calculations. In Fig. $4 \mathrm{~b}$, we plot reaction energies for hydride transfer from the para-substituted BAs to $\mathrm{Au}_{35}$ as a function of cluster charge. Two primary conclusions are apparent from this plot. First, as the cluster becomes more negatively charged, the hydride transfer reaction becomes less favourable. Given the BEP relationship in Fig. 4a, the reaction kinetics also slow down as the cluster becomes more electron-rich. Second, the reaction energy difference between the $-\mathrm{CF}_{3}$ and $-\mathrm{OCH}_{3}$ substituted alcohols decreases as the cluster becomes more electron-rich. This energy difference is, essentially, the computational $\rho$ value for each charged cluster. These calculations are entirely consistent with the experimental results. The $\mathrm{Au} / \mathrm{Al}_{2} \mathrm{O}_{3}$ and $\mathrm{Au} / \mathrm{SiO}_{2}$ catalysts exhibit larger $\rho_{\text {obs }}$ values, suggesting greater charge transfer in the RDS and a somewhat later transition state. Conversely, $\mathrm{Au} / \mathrm{TiO}_{2}$ and $\mathrm{Au} / \mathrm{ZnO}$ have smaller $\rho_{\mathrm{obs}}$ values, suggesting an earlier transition state with less charge transfer to the catalyst.

DFT studies: support effects. The weak correlation between the experimental $\rho_{\text {obs }}$ values and the support bandgap suggests that the oxide electronics influence the electron density at the $\mathrm{Au}$ active site. We therefore considered the support to act as a weak electron donor and sought to use the work function $\phi$ as a simple measure of the oxide electron-donating ability. Although literature values for $\mathrm{TiO}_{2}$ and $\mathrm{ZnO}$ are available, we were unable to find reliable values for $\mathrm{Al}_{2} \mathrm{O}_{3}$ and $\mathrm{SiO}_{2}$. Efforts to directly measure $\phi$ by ultraviolet photoelectron spectroscopy (UPS) were also unsuccessful.

We therefore used DFT to investigate potential relationships between $\rho$ and the oxide work function $\phi$. Because amorphous supports were used in the experimental measurements, we calculated $\phi$ for several surface facets of each oxide support (Supplementary section 'Computational data'), using these calculations as estimates 

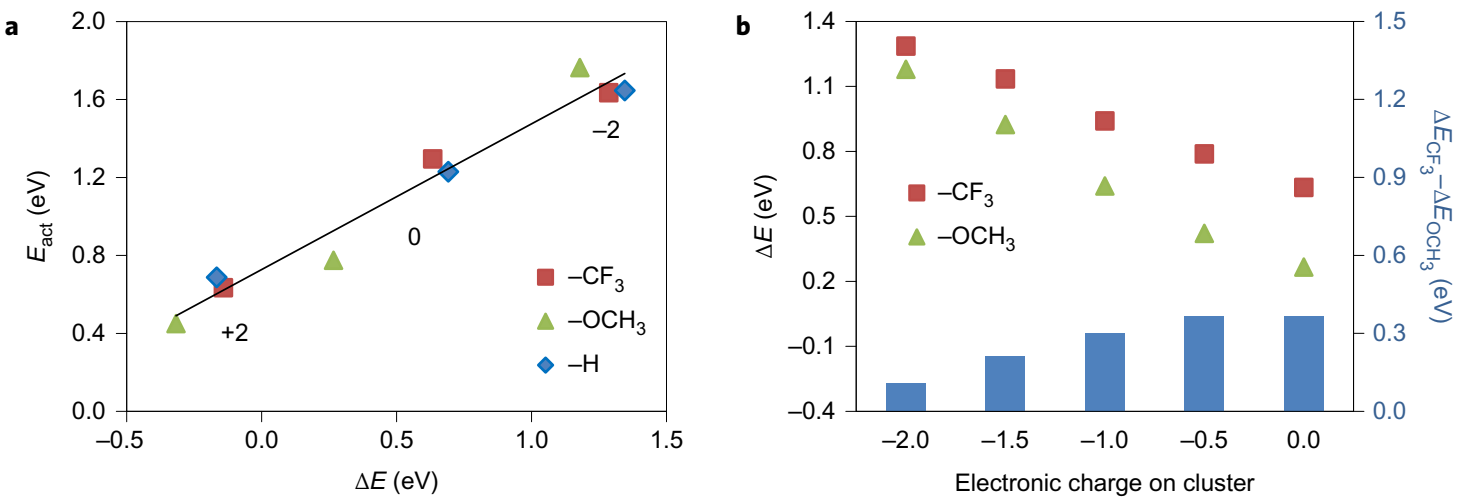

Figure 4 | DFT results. a, BEP relationship between the activation barrier and reaction energy for benzylic hydride transfer from $\mathrm{BA}_{1}$, para-CF $3 \mathrm{BA}$ and para- $\mathrm{OCH}_{3} \mathrm{BA}$ to charged $(-2, \mathrm{O}$ and +2$) \mathrm{Au}_{35}$ nanoparticles. The linear correlation allows us to use the computationally inexpensive reaction energies as a proxy for differences in reaction kinetics. $\mathbf{b}$, Reaction energies for hydride transfer from $-\mathrm{CF}_{3}$ and $-\mathrm{OCH}_{3}$ substituted $\mathrm{BA}$ to $\mathrm{Au}_{35}$ nanoparticles as a function of particle charge. Blue bars at the bottom show the difference between the $-\mathrm{CF}_{3}$ and $-\mathrm{OCH}_{3}$ reaction energies (right $y$ axis), which represents a computational estimate of the Hammett slope $\rho$. DFT studies indicate that, as the cluster becomes more negatively charged, the reaction becomes less favourable (and therefore slower), while the $\rho$ value simultaneously decreases.

for the range of $\phi$ values. For $\mathrm{ZnO}$ and $\mathrm{TiO}_{2}$, the DFT calculations were both $\sim 1.5 \mathrm{eV}$ greater than experimentally determined literature values $(4.3 \pm 0.4 \mathrm{eV} \text { and } 5.2 \pm 0.6 \mathrm{eV} \text {, respectively })^{38}$, which also had a relatively wide range. This indicates that, although there may be some differences between the calculated and experimental values, the calculated trends in work function are likely to be consistent with trends for the real materials.

Figure 5 shows a correlation between the experimentally determined Hammett slopes $\rho_{\text {obs }}$ and DFT-calculated work functions of the metal oxides. In this plot, the width of the bars represents the range of calculated $\phi$ values, and the height represents the experimental error in $\rho_{\text {obs }}$. The relatively large range in $\phi$ for each oxide suggests the electron-donating ability of the support is sensitive to the local environment in which an individual nanoparticle resides. Given the range in $\phi$ and the comparison with $\rho_{\text {obs }}$ values determined on amorphous supports, the data show a reasonable correlation between $\rho_{\text {obs }}$ and $\phi$. Thus, the electron-donation ability of the support, which is a relatively subtle factor, has a comparatively large influence on BA oxidation catalytic activity.

\section{Discussion}

Improving supported metal catalysts requires understanding the key factors that affect active site reactivity; however, few techniques directly probe active-site electronics during catalysis. Hammett studies are uniquely suited to probe subtle differences in electronics during a reaction and therefore offer a unique opportunity to characterize the catalyst active-site electronics. Although they correlate with catalytic activity (Fig. 3a), the experimentally determined $\rho_{\text {obs }}$ values are not direct measures of catalytic activity. Rather, $\rho_{\text {obs }}$ describes how subtle differences in substrate electronics affect the intrinsic reactivity between the substrate and the active site. Thus, $\rho_{\mathrm{obs}}$ is related to the fundamental rate constant of the RDS, whereas catalytic activity depends both on this fundamental reactivity, any preceding steps and the total number of active sites on the catalyst. This is an important distinction because $\rho_{\text {obs }}$ values are independent of the number of active sites for a given catalyst.

This study provides a theoretical foundation for explaining experimentally measured differences in $\rho_{\text {obs }}$ due to the various supports used: stabilizing the transfer of a negatively charged hydride to the Au active sites becomes increasingly difficult as the Au becomes more negatively (or less positively) charged. Correlating this metric with the experimental data, the logical conclusion is that the less active catalysts $\left(\mathrm{Au} / \mathrm{TiO}_{2}\right.$ and $\left.\mathrm{Au} / \mathrm{ZnO}\right)$ have more negatively charged $\mathrm{Au}$ active sites while the more active catalysts $\left(\mathrm{Au} / \mathrm{Al}_{2} \mathrm{O}_{3}\right.$ and $\mathrm{Au} / \mathrm{SiO}_{2}$ ) are less negatively (or perhaps more positively) charged. Furthermore, the DFT results in Fig. 4b suggest a more negatively charged $\mathrm{Au}$ particle will manifest a lower Hammett slope (computationally, the blue bars in Fig. 4b). This provides internal consistency between the experimental TOF- $\rho_{\text {obs }}$ correlation and the DFT correlations between particle charge, activation barrier and Hammett slope. Collectively, this demonstrates that Hammett studies can be used as an in situ measure of the electron richness of the active site on oxide-supported metal catalysts.

Furthermore, because the experimental $\rho_{\text {obs }}$ values did not correlate well with traditional physico-chemical parameters, applying Hammett studies to nanoparticle catalysts in this fashion provides a new opportunity to measure the important yet subtle property of the electron richness or poorness of the active site during catalysis. We clarify that the electron-donation effects observed in the experimental studies are weak ligand effects and should not be confused with a complete electron transfer (which may be observable

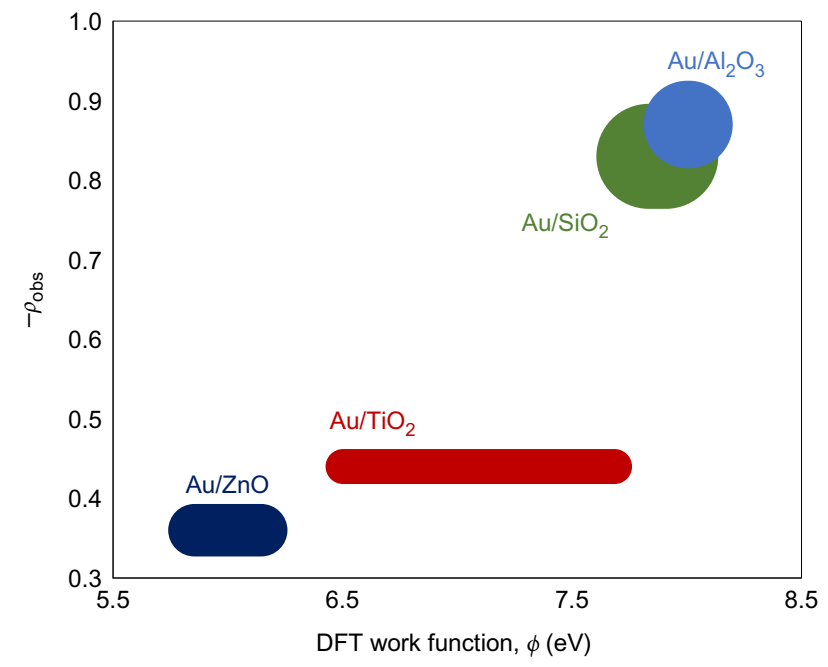

Figure 5 | Correlation between experimentally observed Hammett slopes $\rho_{\text {obs }}$ and DFT-calculated work functions $\phi$. The width of the bars represents the range of $\phi$ values based on the various surfaces used in the calculations (see Supplementary section 'Computational data'), and the height represents the experimental error in $\rho_{\text {obs. }}$. The data show good correlation between $\rho$ for hydride transfer in BA oxidation and the electron-donating ability of the catalyst support, represented by the calculated $\phi$ values. 
with other techniques). Infrared spectroscopy of adsorbed $\mathrm{CO}$ on $\mathrm{Au} / \mathrm{Al}_{2} \mathrm{O}_{3}$ and $\mathrm{Au} / \mathrm{TiO}_{2}$ catalysts shows essentially indistinguishable $\mathrm{CO}$ stretching frequencies $\left(\sim 2,100 \mathrm{~cm}^{-1}\right)$ and adsorption isotherms ${ }^{39}$. This is consistent with the presence of only $\mathrm{Au}^{0}$ sites; there is no indication of either $\mathrm{Au}^{+}$or $\mathrm{Au}^{-}$on either catalyst. This study also shows how the support electronics can affect the catalytic activity of supported metal catalysts. This effect is largely underappreciated; however, the experimental data indicate this electron donation roughly doubles (or halves) the TOF for BA oxidation. We are unaware of any other technique that directly evaluates how the electron richness/poorness of a metal nanoparticle affects catalytic activity.

The purpose of the DFT calculations was to investigate the details of the RDS and identify trends, but physical interpretation of the modelled charges is more complicated. We note that a \pm 2 charge on the cluster seems large, but represents only a \pm 0.06 charge per atom. This is not an unreasonable charge for an $\mathrm{Au}$ atom at or near the metal-support interface, where the interactions with the support are most pronounced and where hydride transfer probably occurs. Because we cannot directly measure the amount of charge transfer, we must limit our interpretations to examining and understanding the trends. Similarly, there may well be morphological differences between Au particles on the different supports. These may be associated with the differences in work function, but we cannot comment further on their roles in affecting reactivity at this stage.

The titania support is somewhat of an outlier from the dominant correlation between $\rho_{\text {obs }}$ and $\phi$, with titania appearing to have a slightly lower than expected $\rho_{\text {obs }}$ value and/or being a somewhat more electron-donating support than is predicted by the DFT calculations. This departure from the trend probably has two origins. First, the range of calculated work functions for titania is more than double that of any of the other oxides, suggesting that local geometries may be particularly important. Second, titania is the most reducible of the supports studied, and oxygen vacancies (which were not included in the DFT calculations) are often implicated in its surface chemistry. The presence of reduced centres on the $\mathrm{TiO}_{2}$ surface would be expected to generate a more electron-rich oxide and hence more electron-rich $\mathrm{Au}$ nanoparticles. Future Hammett studies may provide opportunities to quantify differences in oxygen vacancies as a function of support treatment, or to evaluate the effects of dopants such as $\mathrm{N}$ on the electronics of this important support ${ }^{40}$.

\section{Methods}

Details are available in the Supplementary Information. We performed catalysis experiments in a stirred glass batch reactor thermostatted at $60^{\circ} \mathrm{C}$ under constant ambient $\mathrm{O}_{2}$ pressure. Catalysts were dried at $120^{\circ} \mathrm{C}$ immediately before use, then placed in the reactor with distilled toluene. The reaction was initiated by adding 10 $\mathrm{ml}$ of a $0.10 \mathrm{M}$ BA substrate solution to the reactor. The reaction progress was monitored with gas chromatography, and plots of conversion versus time were used to determine initial reaction rates. Conversions were always held below $10 \%$ and usually below $5 \%$; under these conditions no side products were observed.

Electronic structure calculations were performed using the Vienna ab initio simulation package (VASP) with the Perdew-Wang (PW91) version of the generalized gradient approximation (GGA) exchanged correlation functional ${ }^{41}$. Ion-core electron interactions were represented using the projector augmented-wave (PAW) method, and valence electrons were represented using a plane wave basis set with an energy cutoff of $450 \mathrm{eV}$. A cubic unit cell of size $30 \AA$ was used to calculate hydride abstraction reaction energetics using a Monkhorst Pack $k$-point sampling mesh of $1 \times 1 \times 1$. Forces on all atoms were minimized to $0.05 \mathrm{eV} \AA^{-1}$. Activation barriers were calculated using the climbing image nudged elastic band method $(\mathrm{CI}-\mathrm{NEB})^{42}$. The transition state was identified as the highest-energy image with the tangential force to the reaction coordinate less than $0.05 \mathrm{eV} \AA^{-1}$. Each transition state was confirmed with a vibrational frequency calculation.

Data availability. All data, where not included in the manuscript or Supplementary Information, are available from the authors upon request.

Received 27 October 2016; accepted 15 November 2017; published online 15 January 2018

\section{References}

1. Ponec, V. \& Bond, G. C. in Studies in Surface Science and Catalysis: Catalysis by Metals and Alloys Vol. 95 (eds Delmon, B \& Yates, J. T.) (Elsevier, 1995).

2. Behrens, M. et al. The active site of methanol synthesis over $\mathrm{Cu} / \mathrm{ZnO} / \mathrm{Al}_{2} \mathrm{O}_{3}$ industrial catalysts. Science 336, 893-897 (2012).

3. Daage, M. \& Chianelli, R. R. Structure-function relations in molybdenum sulfide catalysts: the rim-edge model. J. Catal. 149, 414-427 (1994).

4. Chen, M., Kumar, D., Yi, C.-W. \& Goodman, D. W. The promotional effect of gold in catalysis by palladium-gold. Science 310, 291-293 (2005).

5. Schauermann, S., Nilius, N., Shaikhutdinov, S. \& Freund, H.-J. Nanoparticles for heterogeneous catalysis: new mechanistic insights. Acc. Chem. Res. 46, 1673-1681 (2013)

6. Campbell, C. T. Catalyst-support interactions: electronic perturbations. Nat. Chem. 4, 597-598 (2012).

7. Liu, Z. et al. Dry reforming of methane on a highly-active $\mathrm{Ni}-\mathrm{CeO}_{2}$ catalyst: effects of metal-support interactions on $\mathrm{C}-\mathrm{H}$ bond breaking. Angew. Chem. Int. Ed. 55, 7455-7459 (2016).

8. Bruix, A. et al. A new type of strong metal-support interaction and the production of $\mathrm{H}_{2}$ through the transformation of water on $\mathrm{Pt} / \mathrm{CeO}_{2}(111)$ and $\mathrm{Pt} / \mathrm{CeO}_{x} / \mathrm{TiO}_{2}(110)$ catalysts. J. Am. Chem. Soc. 134, 8968-8974 (2012).

9. Lykhach, Y. et al. Counting electrons on supported nanoparticles. Nat. Mater. 15, 284-288 (2016).

10. Vayssilov, G. N. et al. Support nanostructure boosts oxygen transfer to catalytically active platinum nanoparticles. Nat. Mater. 10, 310-315 (2011).

11. Mao, M. et al. Metal support interaction in Pt nanoparticles partially confined in the mesopores of microsized mesoporous $\mathrm{CeO}_{2}$ for highly efficient purification of volatile organic compounds. ACS Catal. 6, 418-427 (2016).

12. Yen, H., Seo, Y., Kaliaguine, S. \& Kleitz, F. Role of metal-support interactions, particle size, and metal-metal synergy in CuNi nanocatalysts for $\mathrm{H}_{2}$ generation. ACS Catal. 5, 5505-5511 (2015).

13. Turner, M. et al. Selective oxidation with dioxygen by gold nanoparticle catalysts derived from 55-atom clusters. Nature 454, 981-983 (2008).

14. Kattel, S. et al. $\mathrm{CO}_{2}$ hydrogenation over oxide-supported PtCo catalysts: the role of the oxide support in determining the product selectivity. Angew. Chem. Int. Ed. 55, 7968-7973 (2016).

15. Greeley, J. P. Active site of an industrial catalyst. Science 336, 810-811 (2012).

16. Divins, N. J., Angurell, I., Escudero, C., Perez-Dieste, V. \& Llorca, J. Influence of the support on surface rearrangements of bimetallic nanoparticles in real catalysts. Science 346, 620-623 (2014).

17. Huang, L., Han, B., Xi, Y., Forrey, R. C. \& Cheng, H. Influence of charge on the reactivity of supported heterogeneous transition metal catalysts. ACS Catal. 5, 4592-4597 (2015)

18. Pacchioni, G. Electronic interactions and charge transfers of metal atoms and clusters on oxide surfaces. Phys. Chem. Chem. Phys. 15, 1737-1757.

19. Bamwenda, G. R., Tsubota, S., Nakamura, T. \& Haruta, M. The influence of the preparation methods on the catalytic activity of platinum and gold supported on $\mathrm{TiO}_{2}$ for CO oxidation. Catal. Lett. 44, 83-87 (1997).

20. Hashmi, S. K. \& Hutchings Graham, J. Gold catalysis. Angew. Chem. Int. Ed. 45, 7896-7936 (2006)

21. Stratakis, M. \& Garcia, H. Catalysis by supported gold nanoparticles: beyond aerobic oxidative processes. Chem. Rev. 112, 4469-4506 (2012).

22. Corma, A. \& Serna, P. Chemoselective hydrogenation of nitro compounds with supported gold catalysts. Science 313, 332-334 (2006).

23. Saavedra, J., Doan, H. A., Pursell, C. J., Grabow, L. C. \& Chandler, B. D. The critical role of water at the gold-titania interface in catalytic $\mathrm{CO}$ oxidation. Science 345, 1599-1602 (2014).

24. Widmann, D. \& Behm, R. J. Activation of molecular oxygen and the nature of the active oxygen species for $\mathrm{CO}$ oxidation on oxide supported Au catalysts. Acc. Chem. Res. 47, 740-749 (2014).

25. Ide, M. S. \& Davis, R. J. The important role of hydroxyl on oxidation catalysis by gold nanoparticles. Acc. Chem. Res. 47, 825-833 (2014).

26. DellaPina, C., Falletta, E. \& Rossi, M. Update on selective oxidation using gold Chem. Soc. Rev. 41, 350-369 (2012).

27. Corma, A. \& Garcia, H. Supported gold nanoparticles as catalysts for organic reactions. Chem. Soc. Rev. 37, 2096-2126 (2008).

28. Xu, B., Madix, R. J. \& Friend, C. M. Predicting gold-mediated catalytic oxidative-coupling reactions from single crystal studies. Acc. Chem. Res. 47, 761-772 (2014)

29. Grirrane, A., Corma, A. \& Garcia, H. Gold-catalyzed synthesis of aromatic azo compounds from anilines and nitroaromatics. Science 322, 1661-1664 (2008).

30. Abad, A., Concepcion, P., Corma, A. \& Garcia, H. A collaborative effect between gold and a support induces the selective oxidation of alcohols. Angew. Chem. Int. Ed. 44, 4066-4069 (2005)

31. Ishida, T., Nagaoka, M., Akita, T. \& Haruta, M. Deposition of gold clusters on porous coordination polymers by solid grinding and their catalytic activity in aerobic oxidation of alcohols. Chem. Eur. J. 14, 8456-8460 (2008)

32. Abad, A., Corma, A. \& Garcia, H. Catalyst parameters determining activity and selectivity of supported gold nanoparticles for the aerobic oxidation of alcohols: the molecular reaction mechanism. Chem. Eur. J. 14, 212-222 (2008). 
33. Fristrup, P., Johansen, L. B. \& Christensen, C. H. Mechanistic investigation of the gold-catalyzed aerobic oxidation of alcohols. Catal. Lett. 120, 184-190 (2008).

34. Conte, M., Miyamura, H., Kobayashi, S. \& Chechik, V. Spin trapping of Au-H intermediate in the alcohol oxidation by supported and unsupported gold catalysts. J. Am. Chem. Soc. 131, 7189-7196 (2009).

35. Hammett, L. P. The effect of structure upon the reactions of organic compounds. Benzene derivatives. J. Am. Chem. Soc. 59, 96-103 (1937).

36. Anslyn, E. V. \& Dougherty, D. A. Modern Physical Organic Chemistry (University Science, 2006).

37. Panthi, B. et al. Using thiol adsorption on supported Au nanoparticle catalysts to evaluate $\mathrm{Au}$ dispersion and the number of active sites for benzyl alcoho oxidation. ACS Catal. 5, 2232-2241 (2015).

38. Henrich, V. E. \& Cox, P. A. The Surface Science of Metal Oxides (Cambridge Univ. Press, 1994).

39. Pursell, C. J., Chandler, B. D., Manzoli, M. \& Boccuzzi, F. CO adsorption on supported gold nanoparticle catalysts: application of the Temkin model. J. Phys Chem. C 116, 11117-11125 (2012).

40. Livraghi, S. et al. Origin of photoactivity of nitrogen-doped titanium dioxide under visible light. J. Am. Chem. Soc. 128, 15666-15671 (2006)

41. Kresse, G. \& Furthmuller, J. Efficient iterative schemes for $a b$ initio total-energy calculations using a plane-wave basis set. Phys. Rev. B 54, 11169-11186 (1996).

42. Henkelman, G., Uberuaga, B. P. \& Jonsson, H. A climbing image nudged elastic band method for finding saddle points and minimum energy paths. J. Chem. Phys. 113, 9901-9904 (2000).

43. Parks, G. A. The isoelectric points of solid oxides, solid hydroxides, and aqueous hydroxo complex systems. Chem. Rev. 65, 177-198 (1965).

\section{Acknowledgements}

The authors thank J.B. Miller and A. Gellman at Carnegie Mellon University for their attempts to measure oxide work functions and S.L.J. Lau at Pennsylvania State University for assistance with work function calculations. The authors acknowledge the US National Science Foundation (NSF, grant nos. CBET-1160217 and CHE-1012395) for financial support of this work. G.K. and M.J.J. acknowledge support from NSF DMREF grant no. 1436206. This work used the Extreme Science and Engineering Discovery Environment (XSEDE), which is supported NSF grant no. ACI-1053575. A.M. and R.M.R. acknowledge the Department of Energy, Office of Basic Energy Sciences, Chemical Sciences, Geosciences and Biosciences Division, Catalysis Sciences Program (grant no. DE-SC0016192) for partial funding of this research. L.T. thanks the Robert A. Welch Foundation (departmental grant no. W-0031) for summer support.

\section{Author contributions}

L.T., J.N., B.P., B.D.C. and C.J.P. designed the catalysis experiments. L.T. and J.N. performed the catalysis experiments and analysed the data. B.P. prepared the $\mathrm{Au} / \mathrm{SiO}_{2}$ catalyst and performed the thiol poisoning experiments. A.M. designed the transmission electron microscopy (TEM) and XPS experiments. A.M. and R.M.R. performed the TEM and XPS experiments and analysed data. G.K. and M.J. designed the computational work. G.K. built the $\mathrm{Au}$ /support model and calculated the BA reaction energetics. G.K. calculated the oxide work functions. B.D.C., G.K. and M.J. wrote the paper with assistance from C.J.P. and R.M.R.

\section{Additional information}

Supplementary information is available in the online version of the paper. Reprints and permissions information is available online at www.nature.com/reprints. Publisher's note: Springer Nature remains neutral with regard to jurisdictional claims in published maps and institutional affiliations. Correspondence and requests for materials should be addressed to B.D.C

\section{Competing financial interests}

The authors declare no competing financial interests. 